Paper

\title{
Bifurcation analysis of inhibitory responses of A PWC spiking neuron model
}

\author{
Yutaro Yamashita ${ }^{1 a)}$ and Hiroyuki Torikai $\left.{ }^{1 b}\right)$ \\ ${ }^{1}$ Graduate School of Engineering Science, Osaka University \\ 1-3 Machikaneyama, Toyonaka, Osaka, Japan \\ a)yutaro@hopf.sys.es.osaka-u.ac.jp \\ b) torikai@sys.es.osaka-u.ac.jp
}

Received April 2, 2012; Revised May 29, 2012; Published October 1, 2012

\begin{abstract}
A piece-wise constant (ab. PWC) spiking neuron model (ab. PWN) has a PWC vector field with a state-dependent reset and is designed to reproduce responses of neurons. Based on analysis techniques for discontinuous ODEs, the dynamics of inhibitory responses of the PWN can be reduced into an one-dimensional iterative map analytically. Using the map, it is shown that the PWN can reproduce bifurcation scenarios of inhibitory responses, which are qualitatively similar to those of the Izhikevich model. In addition, a typical bifurcation scenario can be observed in an actual hardware.
\end{abstract}

Key Words: spiking neuron model, bifurcation, piece-wise constant vector field, VLSI neuron

\section{Introduction}

Neurons exhibit various responses depending on stimulation inputs and parameter values. Many mathematical neuron models and related analog circuit implementations have been studied intensively, where most models are described by continuous ordinary differential equations (ab. ODEs) with/without state-dependent resets, and piece-wise smooth ODEs with/without resets [1-13]. Recently, an alternative hardware-oriented neuron modeling approach by using a piece-wise constant (ab. PWC) ODE with a state-dependent reset has been proposed [14-16]. It has been confirmed that the PWC spiking neuron model (ab. PWN) can reproduce various neuron-like responses like spikings or burstings. Bifurcation phenomena of excitatory responses (i.e., spikings or burstings with respect to increasing stimulation inputs) of the PWN have been analyzed, however, bifurcation phenomena of inhibitory responses (i.e., spikings or burstings with respect to decreasing stimulation inputs) of the PWN have not yet. In this paper, we analyze inhibitory-induced spiking and bursting of the PWN as well as their related bifurcations. First, a circuit model of the PWN is introduced and its neuron-like responses are shown. Second, based on analysis techniques for discontinuous ODEs [17], the dynamics of inhibitory responses can be reduced into an one-dimensional iterative map analytically. Using the map, it is shown that the PWN can reproduce bifurcation scenarios of inhibitory responses, which are qualitatively similar to those of the Izhikevich model. Third, it is confirmed that the bifurcation 
Table I. Comparison between this paper and previous works on the PWN.

\begin{tabular}{|l|c|c|c|}
\hline Bifurcation analysis and experimental measurements of & {$[14]$} & {$[15,16]$} & This paper \\
\hline $\begin{array}{l}\text { excitatory spikings } \\
\text { (i.e., spikings with respect to increasing stimulation inputs) }\end{array}$ & Yes & Yes & - \\
\hline $\begin{array}{l}\text { excitatory burstings } \\
\text { (i.e., burstings with respect to increasing stimulation inputs) }\end{array}$ & - & Yes & - \\
\hline $\begin{array}{l}\text { inhibitory spikings } \\
\text { (i.e., spikings with respect to decreasing stimulation inputs) }\end{array}$ & - & - & Yes \\
\hline $\begin{array}{l}\text { inhibitory burstings } \\
\text { (i.e., burstings with respect to decreasing stimulation inputs) }\end{array}$ & - & - & Yes \\
\hline
\end{tabular}

scenario can be observed in an actual hardware.

Novelties and significances of this paper include the following points. (a) As shown in Table 1, theoretical bifurcation analysis and experimental measurements of excitatory spikings of the PWN have been shown [14]. Also, theoretical bifurcation analysis and experimental measurements of excitatory burstings of the PWN have been shown $[15,16]$. On the other hand, in this paper, theoretical bifurcation analysis and experimental measurements of inhibitory spikings and burstings of the PWN are shown for the first time. Although the PWN can be regarded as a generalized version of PWC oscillators in [18-20], the oscillator is designed as an abstract chaotic oscillator and cannot exhibit neuron-like responses. (b) The subthreshold dynamics of the PWN has a PWC vector field with piece-wise linear switching borders, where such dynamics can be controlled in an easy way by virtue of resistivity of its circuitry to fabrication variation. Hence, the PWN can be regarded as simplification of the Izhikevich model that can be implemented by an electronic circuit with an easy parameter control capability.

\section{Piece-wise constant spiking neuron model}

\subsection{Circuit model}

A piece-wise constant spiking neuron model (ab. PWN) [14-16] is shown in Fig. 1(a). The PWN consists of two capacitors whose capacitances are $C$ and $C$, two voltage-controlled current sources (ab. VCCSs) which are described by functions $I_{v}$ and $I_{u}$, a state-dependent switch $S W$ with an internal resistor $r_{\varepsilon}$, a voltage source $V_{B}$, and an output $Y$. From a neuron model's viewpoint, the capacitor voltages $v$ and $u$ can be regarded as a membrane potential and a recovery variable [1], respectively, as explained in the table in Fig. 1. Figure 1 (b) shows characteristics of each VCCS: it outputs a constant current if the control voltage $v_{\varepsilon}$ is positive and outputs another constant current if $v_{\varepsilon}$ is negative, where the circuit dynamics for $v_{\varepsilon}=0$ is explained later in Section 2.2. If the membrane potential $v$ is below a constant voltage $V_{T}$, the state-dependent switch $S W$ is opened. From a neuron model's viewpoint, the constant voltage $V_{T}$ can be regarded as a spiking threshold. If the membrane potential $v$ reaches the spiking threshold $V_{T}$, the switch $S W$ is closed for a short time duration $t_{\varepsilon}$ and is opened again. In this paper, the time duration $t_{\varepsilon}$ is assumed to be much shorter than $C\left(V_{T}-V_{B}\right) / I_{v}^{+}$and $C\left(V_{T}-V_{B}\right) / I_{v}^{-}$, and the time constant $r_{\varepsilon} C$ is assumed to be much shorter than the time duration $t_{\varepsilon}$. Under these assumptions, the membrane potential $v$ is approximated to exhibit an instantaneous jump to the constant voltage $V_{B}$ when $v$ reaches the spiking threshold $V_{T}$, where $V_{B}$ is referred to as a reset base in this paper. When the switch $S W$ is opened (closed), the PWN outputs a constant voltage $Y=E_{L}$ (an instantaneous pulse $Y=E_{H}$ ). From a neuron model's viewpoint, the pulse $Y=E_{H}$ can be regarded as a firing spike or an action potential as explained in the table in Fig. 1. Also, the PWN accepts a voltage input $V_{i n}$ that can be regarded as a stimulation input. As a result, the dynamics of the PWN is described by the following equation.

$$
\begin{cases}C \dot{v}=I_{v}\left(|v|+V_{i n}-u\right) & \text { if } v<V_{T}, \\ C \dot{u}=I_{u}(a v-u) & \text { if } v(t)=V_{T}, \\ v\left(t^{+}\right)=V_{B} & \text {. }\end{cases}
$$




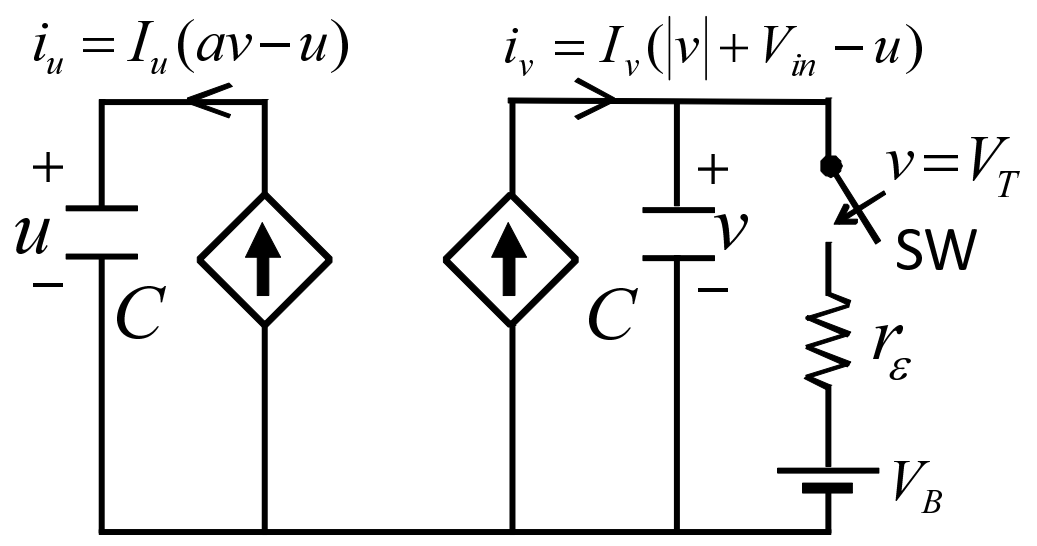

(a)
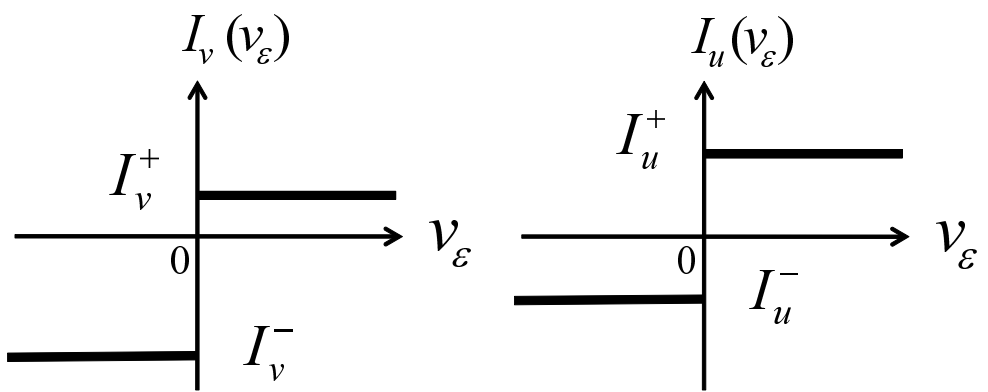

(b)

\begin{tabular}{|l|l|}
\hline PWC spiking neuron & Meaning as a neuron model \\
\hline \hline Capacitor voltage $v$ & Membrane potential \\
\hline Capacitor voltage $u$ & Recovery variable \\
\hline Constant voltage $V_{T}$ & Spiking threshold \\
\hline Pulse $Y=E_{H}$ & Firing spike (Action potential) \\
\hline Voltage input $V_{i n}$ & Stimulation input \\
\hline
\end{tabular}

Fig. 1. (a) The piece-wise constant spiking neuron model (ab. PWN). (b) Characteristics of the voltage-controlled current source (ab. VCCS).

$$
\begin{gathered}
I_{v}\left(v_{\varepsilon}\right)=\left\{\begin{array}{lll}
I_{v}^{+} & \text {if } & v_{\varepsilon}>0, \\
I_{v}^{-} & \text {if } & v_{\varepsilon}<0,
\end{array}\right. \\
I_{u}\left(v_{\varepsilon}\right)=\left\{\begin{array}{lll}
I_{u}^{+} & \text {if } & v_{\varepsilon}>0, \\
I_{u}^{-} & \text {if } & v_{\varepsilon}<0,
\end{array}\right. \\
Y(t)=\left\{\begin{array}{lll}
E_{H} & \text { if } & v(t)=V_{T}, \\
E_{L} & \text { if } & v(t)<V_{T},
\end{array}\right.
\end{gathered}
$$

where the dot "" represents the time derivative " $d / d t$ ", the symbol " $t+$ " represents the moment $" \lim _{\varepsilon \rightarrow+0}(t+\varepsilon)$ " just after $t$ hereafter,

$$
a, V_{B}, I_{v}^{+}, I_{v}^{-}, I_{u}^{+}, I_{u}^{-}
$$

are parameters, $V_{T}>V_{B}$ and $v(0)<V_{T}$ are assumed, and the spiking threshold $V_{T}$ can be normalized to $V_{T}=1$ without loss of generality. $E_{H}$ and $E_{L}$ have no effects against the dynamics of the PWN.

\subsection{Basic dynamics and neuron-like behaviors}

Due to the state-dependent switch $S W$, the state vector $\boldsymbol{v} \equiv(v, u)$ is restricted in the following state space.

$$
\boldsymbol{S} \equiv\left\{\boldsymbol{v} \mid v \leq V_{T}\right\},
$$

where "三" represents the "definition" hereafter. The state space $\boldsymbol{S}$ is divided into the following seven objects. 


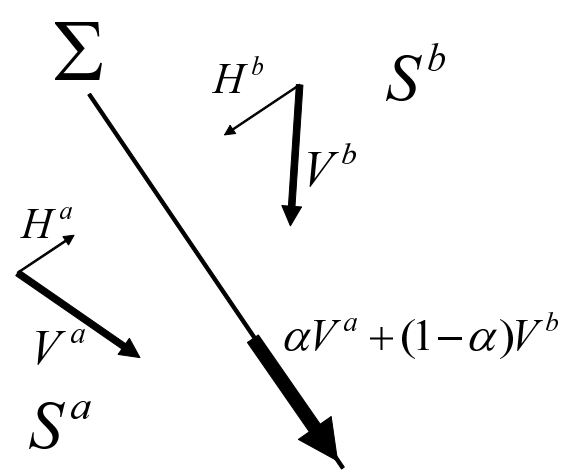

(a) $H^{a}>0$ and $H^{b} \leq 0$.

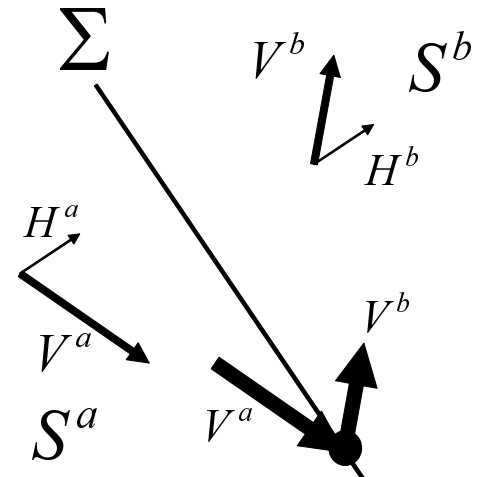

(c) $H^{a}>0$ and $H^{b}>0$.

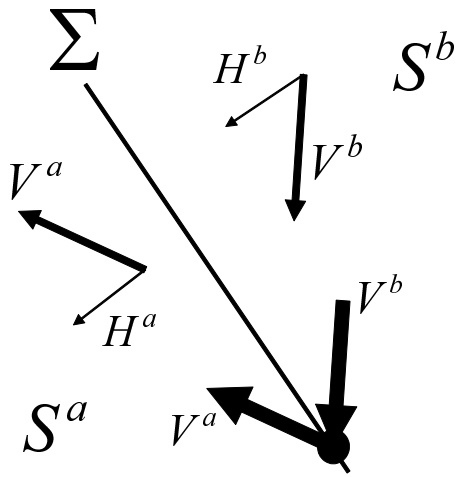

(b) $H^{a} \leq 0$ and $H^{b} \leq 0$.

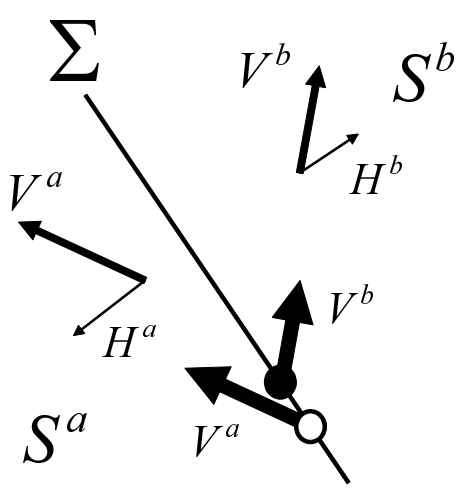

(d) $H^{a} \leq 0$ and $H^{b}>0$.

Fig. 2. Filippov's convex method [17].

Sub-spaces : $\boldsymbol{S}^{++} \equiv\left\{\boldsymbol{v}|u<| v \mid+V_{i n}, u<a v\right.$, and $\left.v<V_{T}\right\}$, $\boldsymbol{S}^{-+} \equiv\left\{\boldsymbol{v}|u>| v \mid+V_{i n}, u<a v\right.$, and $\left.v<V_{T}\right\}$ $\boldsymbol{S}^{+-} \equiv\left\{\boldsymbol{v}|u<| v \mid+V_{\text {in }}, u>a v\right.$, and $\left.v<V_{T}\right\}$, $\boldsymbol{S}^{--} \equiv\left\{\boldsymbol{v}|u>| v \mid+V_{\text {in }}, u>a v\right.$, and $\left.v<V_{T}\right\}$

$v$-border : $\quad \boldsymbol{\Sigma}_{v} \equiv\left\{\boldsymbol{v}|u=| v \mid+V_{\text {in }}\right.$ and $\left.v<V_{T}\right\}$,

$u$-border : $\quad \boldsymbol{\Sigma}_{u} \equiv\left\{\boldsymbol{v} \mid u=a v\right.$ and $\left.v<V_{T}\right\}$,

Spiking threshold: $\boldsymbol{T} \equiv\left\{\boldsymbol{v} \mid v=V_{T}\right\}$.

Note that the sub-spaces $\left(\boldsymbol{S}^{++}, \boldsymbol{S}^{-+}, \boldsymbol{S}^{+-}, \boldsymbol{S}^{--}\right)$correspond to the case of $v_{\varepsilon} \neq 0$ for the VCCSs $I_{v}\left(v_{\varepsilon}\right)$ and $I_{u}\left(v_{\varepsilon}\right)$, and that the borders $\boldsymbol{\Sigma}_{v}$ and $\boldsymbol{\Sigma}_{u}$ correspond to the cases of $v_{\varepsilon}=0$ for the VCCSs $I_{v}\left(v_{\varepsilon}\right)$ and $I_{u}\left(v_{\varepsilon}\right)$, respectively. The dynamics of the state vector $\boldsymbol{v}=(v, u)$ on the sub-spaces $\left(\boldsymbol{S}^{++}, \boldsymbol{S}^{-+}, \boldsymbol{S}^{+-}, \boldsymbol{S}^{--}\right)$and the spiking threshold $\boldsymbol{T}$ is described by the following equations.

Dynamics on the sub-spaces $\left(S^{++}, S^{-+}, S^{+-}, S^{--}\right)$:

$$
C \dot{\boldsymbol{v}}= \begin{cases}\boldsymbol{V}^{++} \equiv\left(I_{v}^{+}, I_{u}^{+}\right) & \text {if } \boldsymbol{v} \in \boldsymbol{S}^{++} \\ \boldsymbol{V}^{-+} \equiv\left(I_{v}^{-}, I_{u}^{+}\right) & \text {if } \boldsymbol{v} \in \boldsymbol{S}^{-+} \\ \boldsymbol{V}^{+-} \equiv\left(I_{v}^{+}, I_{u}^{-}\right) & \text {if } \boldsymbol{v} \in \boldsymbol{S}^{+-} \\ \boldsymbol{V}^{--} \equiv\left(I_{v}^{-}, I_{u}^{-}\right) & \text {if } \boldsymbol{v} \in \boldsymbol{S}^{--}\end{cases}
$$

Dynamics on the spiking threshold $T$ :

$$
\boldsymbol{v}\left(t^{+}\right)=\boldsymbol{B} \equiv\left\{\boldsymbol{v} \mid v=V_{B}\right\} \quad \text { if } \quad \boldsymbol{v}(t) \in \boldsymbol{T} .
$$

The dynamics of the state $\boldsymbol{v}$ on the borders $\boldsymbol{\Sigma}_{v}$ and $\boldsymbol{\Sigma}_{u}$ can be categorized into sliding mode and non-sliding mode (we also say "without sliding mode") [14-17]. Whether the dynamics on each subborder is categorized into the sliding one or the non-sliding one depends on the parameter values in the parameters (2). In order to describe the sliding/non-sliding mode dynamics, the following subsets (we also say "sub-borders") of the borders $\boldsymbol{\Sigma}_{v}$ and $\boldsymbol{\Sigma}_{u}$ can be defined if exist. 


$$
\begin{aligned}
& \boldsymbol{\Sigma}_{v 1} \equiv\left\{\boldsymbol{v} \mid \boldsymbol{v} \in \boldsymbol{\Sigma}_{v}, u<a v, v \leq 0 \text { and } v<V_{T}\right\}, \\
& \boldsymbol{\Sigma}_{v 2} \equiv\left\{\boldsymbol{v} \mid \boldsymbol{v} \in \boldsymbol{\Sigma}_{v}, u<a v, v>0 \text { and } v<V_{T}\right\}, \\
& \boldsymbol{\Sigma}_{v 3} \equiv\left\{\boldsymbol{v} \mid \boldsymbol{v} \in \boldsymbol{\Sigma}_{v}, u>a v, v \leq 0 \text { and } v<V_{T}\right\}, \\
& \boldsymbol{\Sigma}_{v 4} \equiv\left\{\boldsymbol{v} \mid \boldsymbol{v} \in \boldsymbol{\Sigma}_{v}, u>a v, v>0 \text { and } v<V_{T}\right\}, \\
& \boldsymbol{\Sigma}_{u 1} \equiv\left\{\boldsymbol{v}\left|\boldsymbol{v} \in \boldsymbol{\Sigma}_{u}, u>\right| v \mid+V_{i n}, \text { and } v<V_{T}\right\}, \\
& \boldsymbol{\Sigma}_{u 2} \equiv\left\{\boldsymbol{v}\left|\boldsymbol{v} \in \boldsymbol{\Sigma}_{u}, u<\right| v \mid+V_{i n}, \text { and } v<V_{T}\right\} .
\end{aligned}
$$

The dynamics on each sub-border has possibilities to be sliding or non-sliding and thus the dynamics of the state $\boldsymbol{v}$ on the borders $\boldsymbol{\Sigma}_{v}$ and $\boldsymbol{\Sigma}_{u}$ can be categorized into $2^{6}=64$ cases. Let us consider the following general situation as explained in Fig. 2.

- A sub-border $\boldsymbol{\Sigma}$ divides sub-spaces $\boldsymbol{S}^{a}$ and $\boldsymbol{S}^{b}$, where $\boldsymbol{\Sigma} \in\left\{\boldsymbol{\Sigma}_{v 1}, \boldsymbol{\Sigma}_{v 2}, \boldsymbol{\Sigma}_{v 3}, \boldsymbol{\Sigma}_{v 4}, \boldsymbol{\Sigma}_{u 1}, \boldsymbol{\Sigma}_{u 2}\right\}$, and $\boldsymbol{S}^{a}, \boldsymbol{S}^{b} \in\left\{\boldsymbol{S}^{++}, \boldsymbol{S}^{-+}, \boldsymbol{S}^{+-}, \boldsymbol{S}^{--}\right\}$.

- The vector fields on the sub-spaces are

$$
C \dot{\boldsymbol{v}}= \begin{cases}\boldsymbol{V}^{a} & \text { if } \boldsymbol{v} \in \boldsymbol{S}^{a} \\ \boldsymbol{V}^{b} & \text { if } \boldsymbol{v} \in \boldsymbol{S}^{b}\end{cases}
$$

where $\boldsymbol{V}^{a}, \boldsymbol{V}^{b} \in\left\{\boldsymbol{V}^{++}, \boldsymbol{V}^{-+} \boldsymbol{V}^{+-}, \boldsymbol{V}^{--}\right\}$.

We define $H^{a}, H^{b} \in \Re$ as the components of $\boldsymbol{V}^{a}$ and $\boldsymbol{V}^{b}$ which are vertical to the sub-border $\boldsymbol{\Sigma}$, respectively [17]. As shown in Fig. 2, the trajectory of $\boldsymbol{v}$ approaches the sub-border $\boldsymbol{\Sigma}$ if $\boldsymbol{v} \in \boldsymbol{S}^{a}$ and $H^{a}>0$, or $\boldsymbol{v} \in \boldsymbol{S}^{b}$ and $H^{b} \leq 0$. The trajectory of $\boldsymbol{v}$ gets away from the sub-border $\boldsymbol{\Sigma}$ if $\boldsymbol{v} \in \boldsymbol{S}^{a}$ and $H^{a} \leq 0$, or $\boldsymbol{v} \in \boldsymbol{S}^{b}$ and $H^{b}>0$. As a result, the dynamics on each sub-border $\boldsymbol{\Sigma}$ can be described as follows by using Filippov's convex method [17].

Dynamics on each sub-border $\Sigma$ :

$$
C \dot{\boldsymbol{v}}= \begin{cases}(1-\alpha) V^{a}+\alpha V^{b} & \text { if } \quad H^{a}>0 \text { and } H^{b} \leq 0, \\ V^{a} & \text { if } H^{a} \leq 0 \text { and } H^{b} \leq 0, \\ V^{b} & \text { if } H^{a}>0 \text { and } H^{b}>0, \\ V^{b} & \text { if } \quad H^{a} \leq 0 \text { and } H^{b}>0,\end{cases}
$$

where $0<\alpha=-H^{a} /\left(H^{b}-H^{a}\right)<1$. Now the dynamics of the PWN is described by Eqs. (3), (4), and (5). Figure 3 shows typical neuron-like behaviors of the PWN. Let us explain relations between these behaviors and neurons' behaviors.

Resting States (SRS and URS): In the case of Fig. 3(a), the intersection point $\boldsymbol{S}_{0}=\boldsymbol{\Sigma}_{v} \cap \boldsymbol{\Sigma}_{u}$ of the borders attracts any nearby point. From a neuron model's viewpoint, such a phenomenon is called stable resting state (ab. SRS) [1]. In the case of Fig. 3(c), the intersection point $\boldsymbol{S}_{0}=\boldsymbol{\Sigma}_{v} \cap \boldsymbol{\Sigma}_{u}$ repels any nearby point. Such a phenomenon is called an unstable resting state (ab. URS).

Tonic Spikings (STS and UTS): In the case of Fig. 3(b), the state vector $\boldsymbol{v}=(v, u)$ continues to move on a closed orbit periodically. From a neuron model's viewpoint, such a phenomenon is called tonic spiking [1]. If the tonic spiking orbit attracts any nearby point, it is called a stable tonic spiking (ab. STS). If the tonic spiking orbit repels any nearby point, it is called an unstable tonic spiking (ab. UTS).

Tonic Burstings (STB and UTB): In the case of Fig. 3(c), the state vector $\boldsymbol{v}$ repeats the following behaviors periodically: frequently spikes near the spiking threshold $V_{T}$, moves away from the spiking threshold $V_{T}$, and returns into the spiking threshold $V_{T}$. From a neuron model's viewpoint, such a phenomenon is called tonic bursting [1]. If the tonic bursting orbit attracts any nearby point, it is called a stable tonic bursting (ab. STB). If the tonic bursting orbit repels any nearby point, it is called an unstable tonic bursting (ab. UTB). 

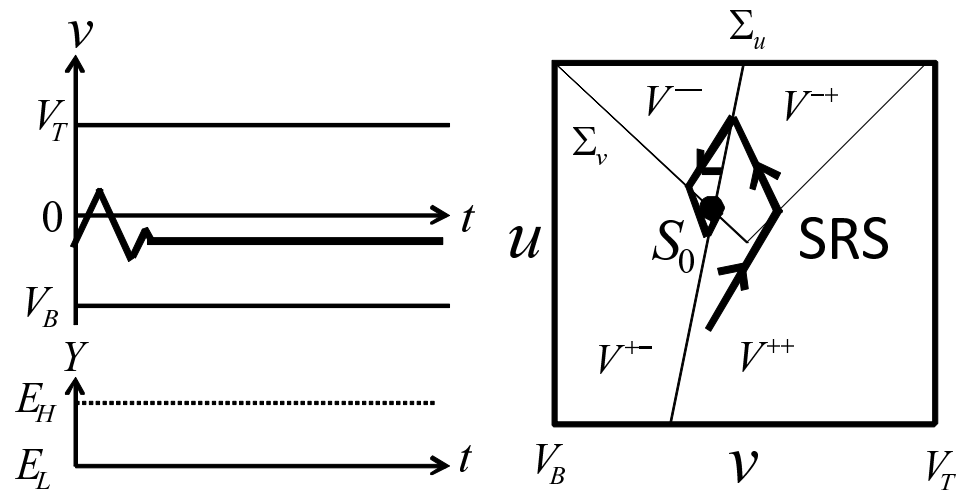

(a) Stable resting state (SRS).
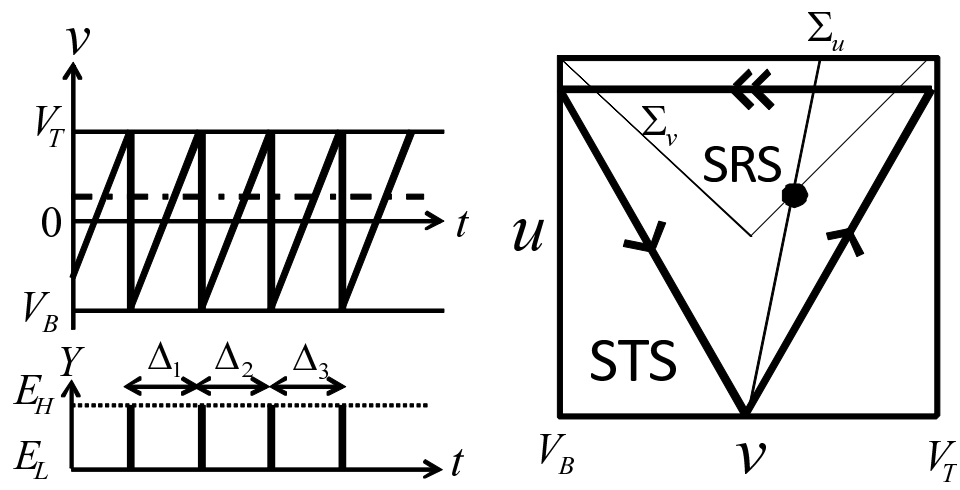

(b) Co-existence of stable resting state (SRS) and stable tonic spiking (STS).
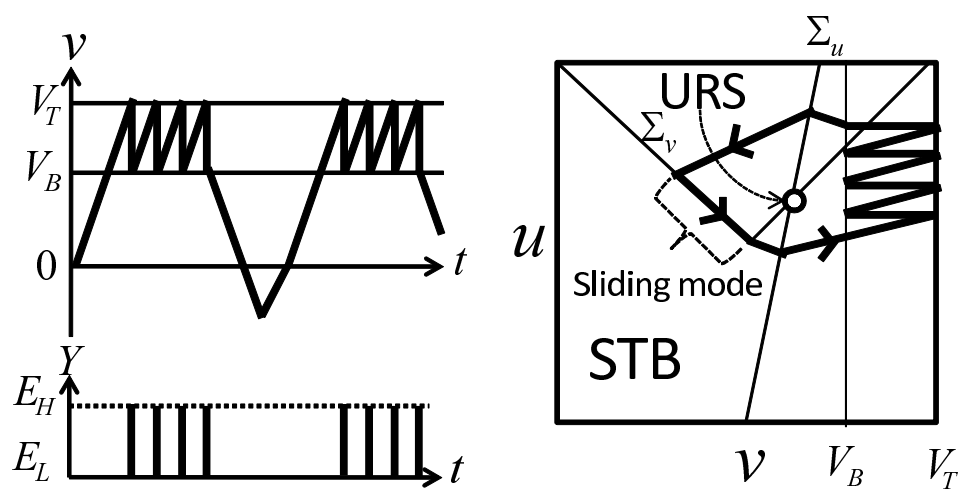

(c) Stable tonic bursting (STB).

Fig. 3. Basic behaviors of the PWN.

\section{Bifurcation analysis of neuron-like inhibitory-induced bursting}

\subsection{Problem statement}

In this section, we study inhibitory responses of the PWN. Figure 4(a) shows a steady state behavior of the PWN and there exists an SRS. In Fig. 4(b), the stimulation input $V_{i n}$ is decreased and there exists an STS. In Fig. 4(c), the stimulation input $V_{i n}$ is further decreased and there exists an STB. A neuron which spikes or bursts as the stimulation input is decreased is said to exhibit inhibitory responses [1]. On the other hand, a neuron which spikes or bursts as the stimulation input is increased is said to exhibit excitatory responses [1]. It is important to study not only excitatory responses but also inhibitory ones, e.g., it is suggested that neural inhibition realizes negative feedbacks in networks of neurons and maintain stabilities of the networks [21]. In the next subsection, we study inhibitory responses and underlying bifurcations of the PWN, where excitatory responses have been analyzed in $[14-16]$. 


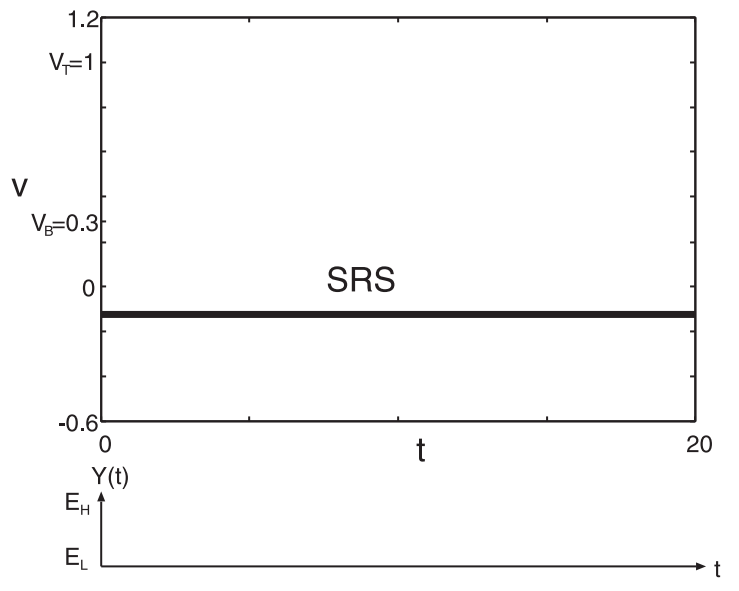

(a) SRS.

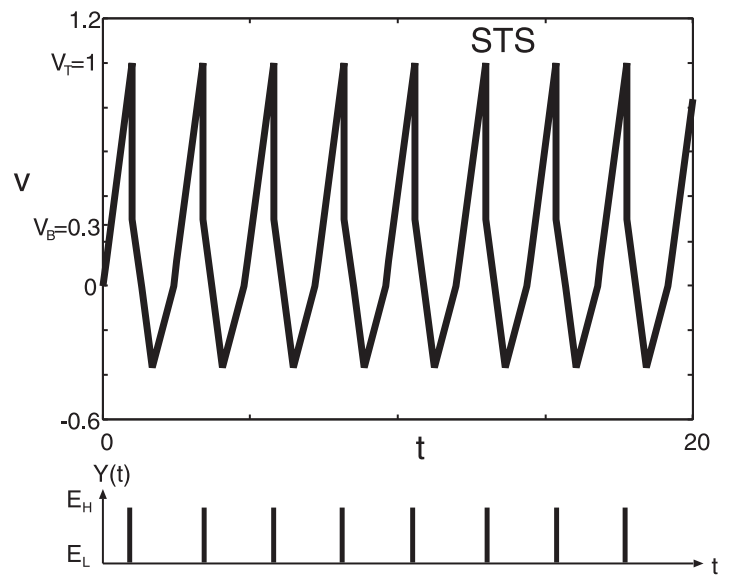

(b) STS.

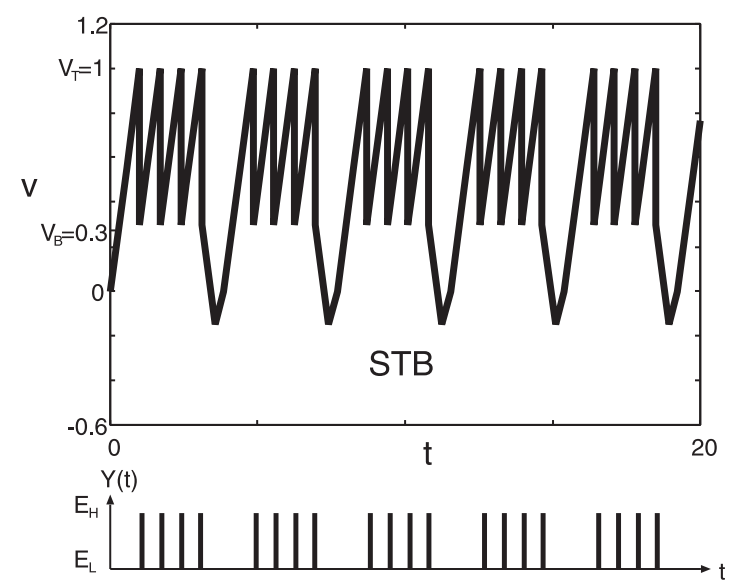

(c) STB.

Fig. 4. Neuron-like responses of the PWN. The parameters of the PWN are $a=-5.0, I_{v}^{+}=1.0, I_{v}^{-}=-1.0, I_{u}^{+}=-0.5, I_{u}^{-}=0.5, V_{T}=1.0, V_{B}=0.3$, and $C=1.0$. (a) Stable resting state (SRS). $V_{i n}=0.5$. (b) Stable Tonic Spiking (STS). $V_{i n}=-0.5$. (c) Stable Tonic Bursting (STB). $V_{i n}=-2.5$.

\subsection{Bifurcation analysis}

In order to analyze the inhibitory responses, the following parameter condition is assumed.

$$
a<-1, I_{v}^{+}=-I_{v}^{-}=1.0,-1<I_{u}^{+}=-I_{u}^{-}<0, V_{T}=1, V_{B}>0 .
$$

Inspired by the vector-field settings of the Izhikevich model [1-3], the parameter condition (6) is determined ${ }^{1}$. In the case of the parameter condition (6), the dynamics of the state vector $\boldsymbol{v}$ on the borders $\boldsymbol{\Sigma}_{v}$ and $\boldsymbol{\Sigma}_{u}$ can be described by the following equation [17].

Dynamics on the $v$-border $\boldsymbol{\Sigma}_{v}$ and $u$-border $\boldsymbol{\Sigma}_{v}$ :

$$
C \dot{\boldsymbol{v}}= \begin{cases}\left(-I_{u}^{+}, I_{u}^{+}\right) & \text {if } \boldsymbol{v} \in \boldsymbol{\Sigma}_{v 1}, \\ \boldsymbol{V}^{++} & \text {if } \boldsymbol{v} \in \boldsymbol{\Sigma}_{v 2}, \\ \left(-I_{u}^{-}, I_{u}^{-}\right) & \text {if } \boldsymbol{v} \in \boldsymbol{\Sigma}_{v 3}, \\ \boldsymbol{V}^{--} & \text {if } \boldsymbol{v} \in \boldsymbol{\Sigma}_{v 4}, \\ \boldsymbol{V}^{-+} & \text {if } \boldsymbol{v} \in \boldsymbol{\Sigma}_{u 1}, \\ \boldsymbol{V}^{+-} & \text {if } \boldsymbol{v} \in \boldsymbol{\Sigma}_{u 2} .\end{cases}
$$

Now we derive an iterative map $F$ as the followings. As shown in Fig. 5(d), let an initial state of $\boldsymbol{v}=$ $(v, u)$ on the spiking threshold $\boldsymbol{T} \equiv\left\{(v, u) \mid v=V_{T}\right\}$ be denoted by $\left(v_{n}, u_{n}\right)$. The point $\left(v_{n}, u_{n}\right) \in \boldsymbol{T}$ can be represented by its $u$-coordinate $u_{n} \in(-\infty,+\infty)$. The trajectory starting from $\left(v_{n}, u_{n}\right)$ returns

\footnotetext{
${ }^{1}$ The parameter condition (6) is inspired by those of inhibitory responses of the Izhikevich model. Under this condition, Eq. (7) realizes the counter-clockwise behaviors of the state vector $(u, v)$ of the PWN.
} 

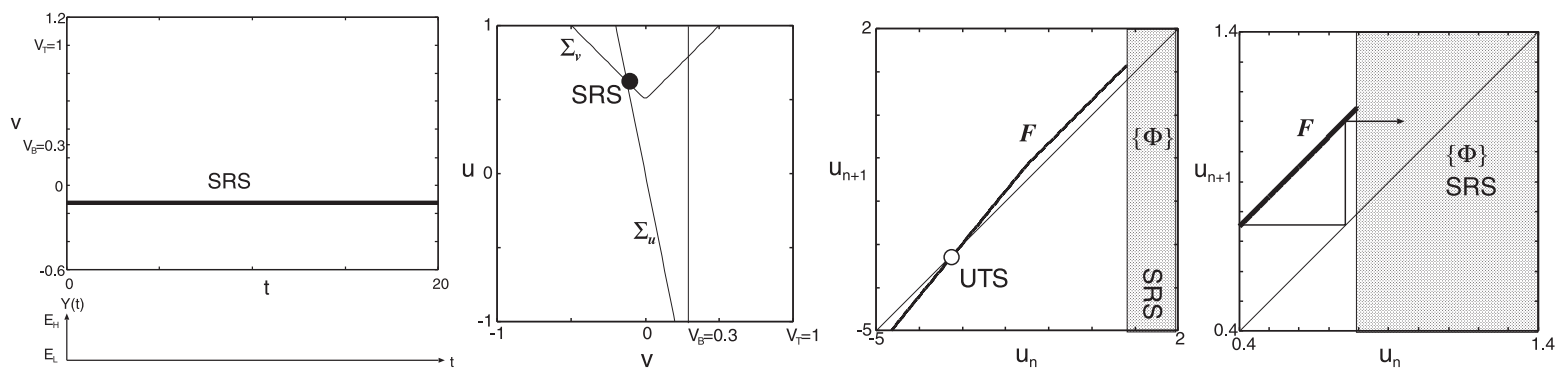

(a)
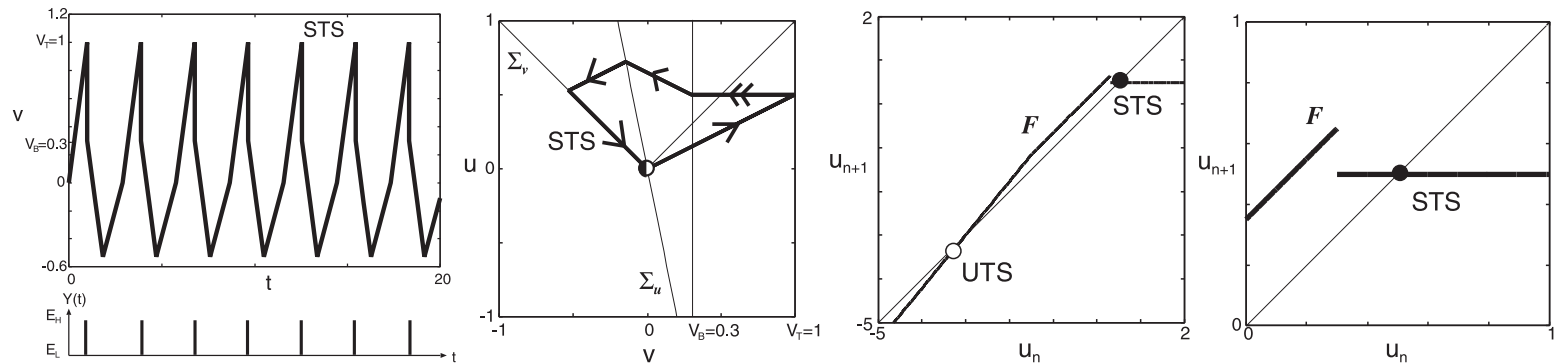

(b)
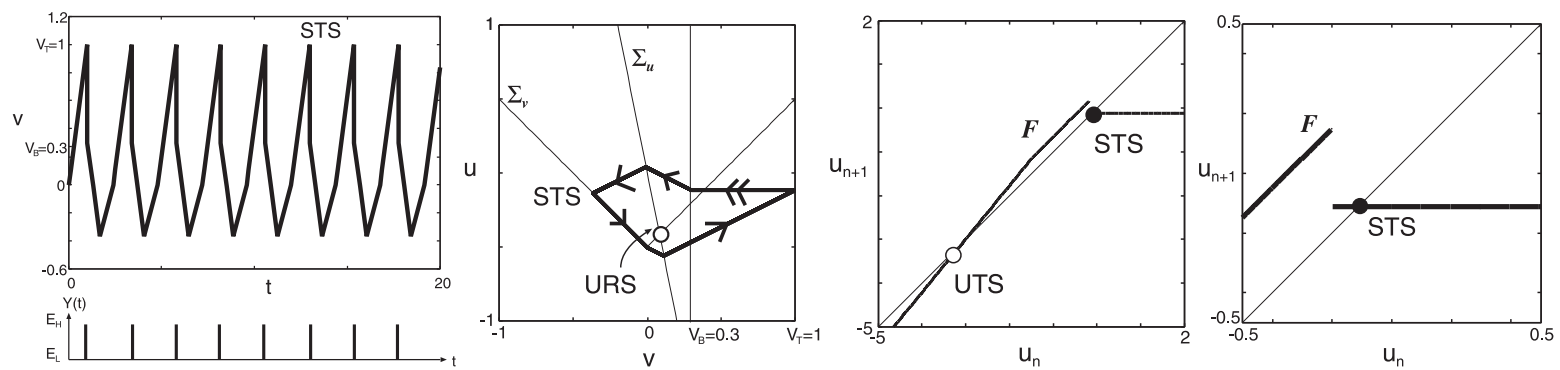

(c)
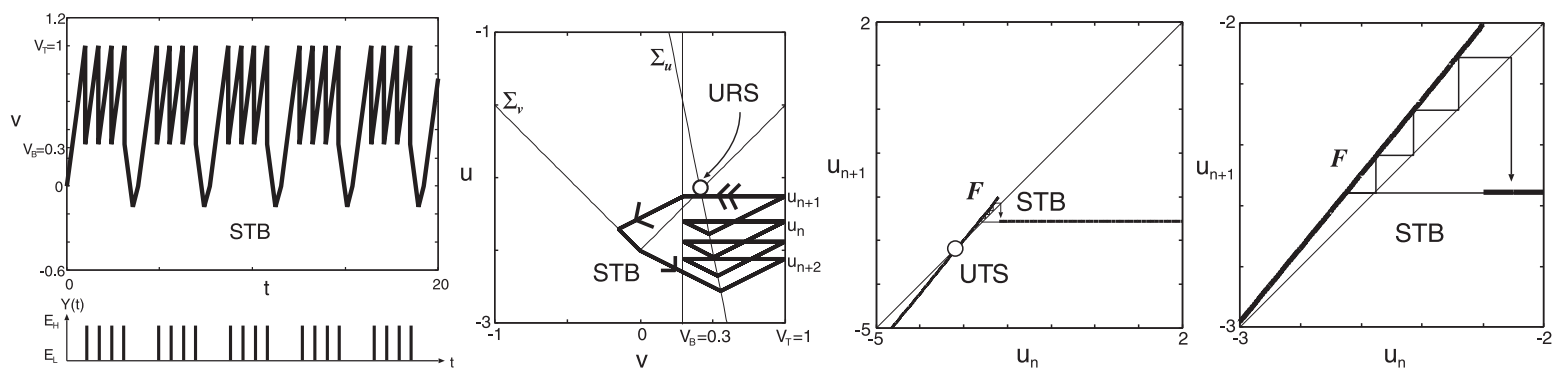

(d)

Fig. 5. The Bifurcation Scenario for $b \geq V_{B}$. The parameters of the PWN are $a=-5.0, I_{v}^{+}=1.0, I_{v}^{-}=-1.0, I_{u}^{+}=-0.5, I_{u}^{-}=0.5$, $V_{T}=1.0, V_{B}=0.3$, and $C=1.0$. (a) Stable resting state (SRS). $V_{i n}=$ 0.5. (b) Stability-changing border-collision bifurcation. $V_{i n}=0.0$. (c) Stable Tonic Spiking (STS). $V_{i n}=-0.5$. (d) Stable Tonic Bursting (STB). $V_{i n}=-2.5$. (e) Fold limit cycle type border-collision bifurcation. $V_{i n}=-2.9$. (f) Divergence. $V_{i n}=-3.0$. (g) Bifurcation diagram. The arrows (a)-(f) correspond to (a)-(f), respectively. 

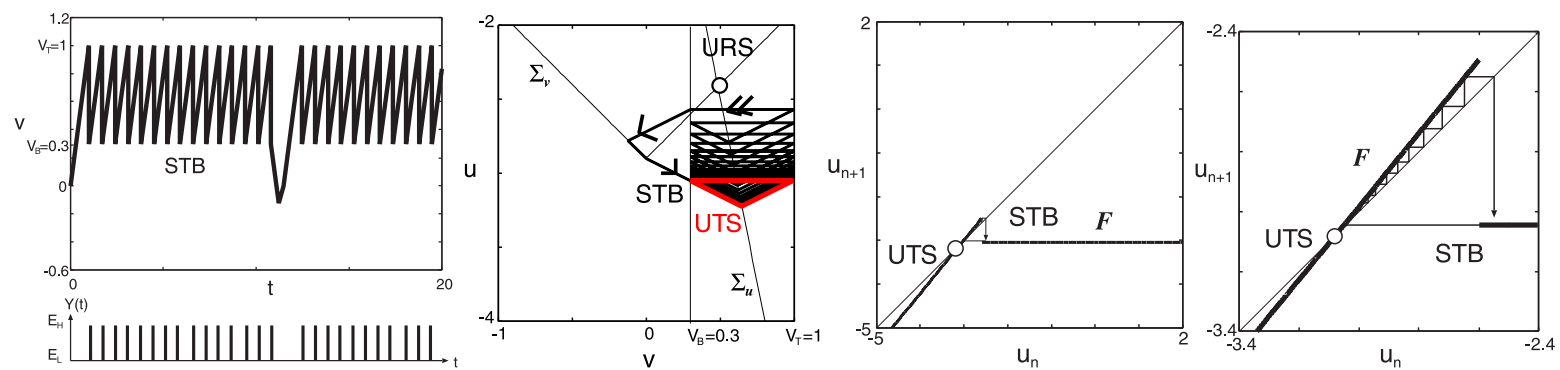

(e)
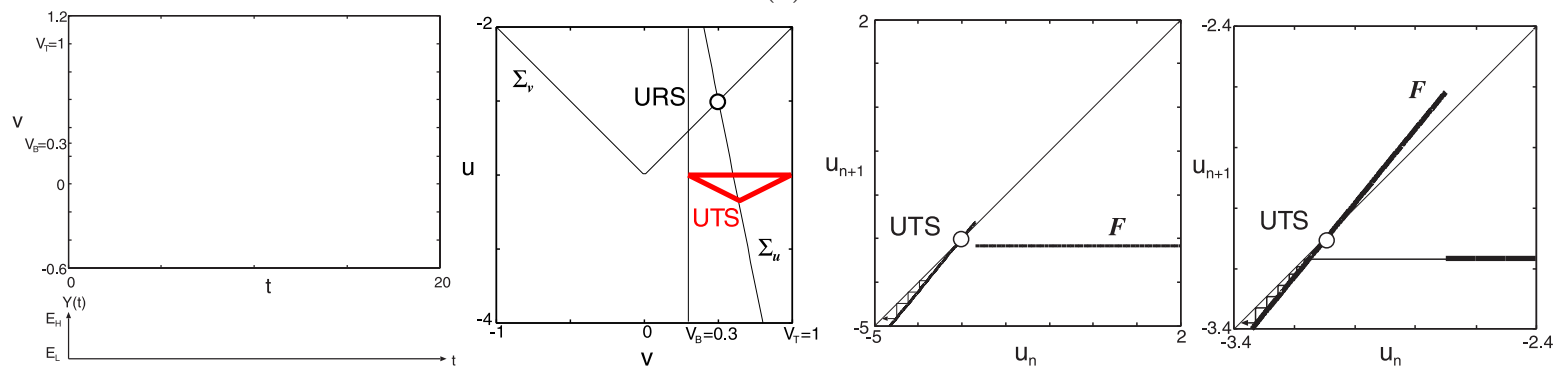

(f)

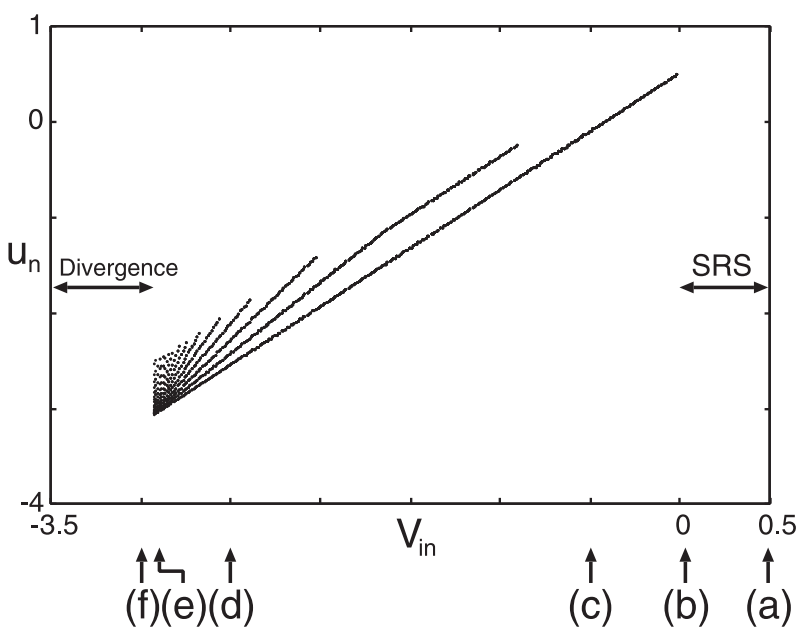

(g)

Fig. 5. (Continued.)

into the spiking threshold $\boldsymbol{T}$ after visiting some of the sub-spaces $\left\{\boldsymbol{S}^{++}, \boldsymbol{S}^{+-}, \boldsymbol{S}^{-+}, \boldsymbol{S}^{--}\right\}$and the sliding vector fields. Let the return point of $\boldsymbol{v}=(v, u)$ on the spiking threshold $\boldsymbol{T}$ be denoted by $\left(v_{n+1}, u_{n+1}\right)$ if it returns. If the trajectory never returns into the spiking threshold $\boldsymbol{T}$, the image of the map $F$ is defined as the empty set $\{\emptyset\}$. Then the dynamics of the state $u_{n}$ is described by the following one-dimensional iterative map $F$ analytically without any approximation (see Fig. 5).

$$
u_{n+1}=F\left(u_{n}\right), \quad F:(-\infty, \infty) \rightarrow(-\infty, \infty) \cup\{\emptyset\},
$$

where $n=1,2,3, \cdots$. In order for a formula of the iterative map $F$ to be simplified, the following parameter condition is assumed.

$$
\left|I_{u}^{+} / I_{v}^{+}\right|=\left|I_{u}^{+} / I_{v}^{-}\right|=\left|I_{u}^{-} / I_{v}^{+}\right|=\left|I_{u}^{-} / I_{v}^{-}\right|=b .
$$

Then the following analytic formula of the iterative map $F$ can be derived. 


$$
\begin{aligned}
& F\left(u_{n}\right)=\left\{\begin{array}{lll}
u_{n}-b\left(V_{T}-V_{B}\right) & \text { if } \quad \begin{array}{l}
V_{i n} \in \overline{\boldsymbol{L}}_{5} \text { and } u_{n} \in \boldsymbol{T}_{1}, \\
\text { or } V_{i n} \in \boldsymbol{L}_{5} \text { and } u_{n} \in \boldsymbol{T}_{3},
\end{array} \\
b V_{T}+\frac{\left(u_{n}+b V_{B}\right)(a-b)}{a+b} & \text { if } \quad \begin{array}{l}
V_{i n} \in \boldsymbol{L}_{1} \cup \boldsymbol{L}_{2} \text { and } u_{n} \in \overline{\boldsymbol{T}}_{1} \cap \boldsymbol{T}_{2}, \\
\text { or } V_{i n} \in \boldsymbol{L}_{3} \cup \boldsymbol{L}_{4} \text { and } u_{n} \in \overline{\boldsymbol{T}}_{1} \cap \boldsymbol{T}_{3},
\end{array} \\
u_{n}+b\left(V_{T}-V_{B}\right) & \text { if } \quad V_{i n} \in \boldsymbol{L}_{1} \cup \boldsymbol{L}_{2} \text { and } u_{n} \in \overline{\boldsymbol{T}}_{2} \cap \boldsymbol{T}_{3}, \\
b V_{T}+\frac{a-b}{a+b} V_{i n} & \text { if } \quad V_{i n} \in \boldsymbol{L}_{2} \cup \boldsymbol{L}_{3} \text { and } u_{n} \in \overline{\boldsymbol{T}}_{3}, \\
-b V_{T}+V_{i n} & \text { if } \quad V_{i n} \in \boldsymbol{L}_{4} \cup \boldsymbol{L}_{5} \text { and } u_{n} \in \overline{\boldsymbol{T}}_{3}, \\
\{\emptyset\} & \text { if } \quad V_{i n} \in \boldsymbol{L}_{1} \text { and } u_{n} \in \overline{\boldsymbol{T}}_{3},
\end{array}\right. \\
& \boldsymbol{L}_{1} \equiv\left\{V_{i n} \mid V_{i n}>0\right\}, \quad \boldsymbol{L}_{2} \equiv\left\{V_{i n} \mid(a-1) V_{B}<V_{i n} \leq 0\right\}, \\
& \boldsymbol{L}_{3} \equiv\left\{V_{i n} \mid(a+b) V_{T}<V_{i n} \leq(a-1) V_{B}\right\}, \\
& \boldsymbol{L}_{4} \equiv\left\{V_{i n} \mid-(1+b) V_{B}+(a+b) V_{T}<V_{i n} \leq(a+b) V_{T}\right\} \text {, } \\
& \boldsymbol{L}_{5} \equiv\left\{V_{i n} \mid V_{i n}<-(1+b) V_{B}+(a+b) V_{T}\right\}, \\
& \boldsymbol{T}_{1} \equiv\left\{u_{n} \mid u_{n}<a V_{T}+b\left(V_{T}-V_{B}\right)\right\} \text {, } \\
& \boldsymbol{T}_{2} \equiv\left\{u_{n} \mid u_{n}<a V_{B}\right\}, \quad \boldsymbol{T}_{3} \equiv\left\{u_{n} \mid u_{n}<V_{B}+V_{i n}\right\},
\end{aligned}
$$

where "«", " $\cap$ ", and "บ" indicate "compliment", "intersection", and "union", respectively.

\subsubsection{Case I $: b \geq V_{B}$}

In Fig. 5(a) that corresponds to Fig. 4(a), there exists an SRS. In Fig. 5(b), the stimulation input $V_{i n}$ is decreased compared to Fig. 5(a), the URS is unstabilized, and an STS is born. In Fig. 5(c) that corresponds to Fig. 4(b), there exists the STS. The change of phenomena between Fig. 5(a) and Fig. 5(c) (i.e., unstabilization of the SRS and birth of the STS) is caused by the discontinuous change of the stability of the resting state and thus we refer to it as a stability-changing border-collision bifurcation. In Fig. 5(d) that corresponds to Fig. 4(c), the stimulation input $V_{i n}$ is decreased compared to Fig. 5(c), and there exists an STB. Judging from the shape of the map $F$, the change of phenomena between Fig. 5(c) and Fig. 5(d) (i.e., change from the STS to the STB) is a typical border-collision bifurcation. In Fig. 5(e), the stimulation input $V_{i n}$ is decreased compared to Fig. 5(d), and the STB vanishes since it is eaten by an UTS. In Fig. $5(\mathrm{f})$, the stimulation input $V_{\text {in }}$ is decreased compared to Fig. 5(e), and the orbit diverges. The change of phenomena between Fig. 5(d) and Fig. 5(f) (i.e., vanish of the STB) has qualitative similarities to the fold limit cycle bifurcation. Hence, we refer to it as a fold limit cycle type border-collision bifurcation. Figure $5(\mathrm{~g})$ shows a bifurcation diagram, where (a)-(f) in Fig. 5(g) correspond to Fig. 5(a)-(f), respectively. As a result, the scenario of the change of the phenomena from/to the SRS in Fig. 5(a) to/from the STS in Fig. 5(f) can be summarized as follows.

The Bifurcation Scenario for $b \geq V_{B}$ :

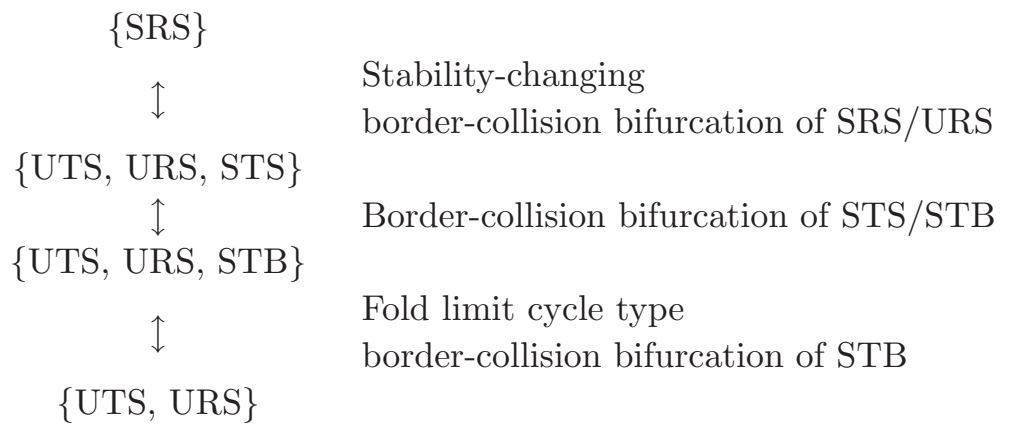

Neuron-likeness of this bifurcation scenario is discussed in the subsection 3.2.3. 


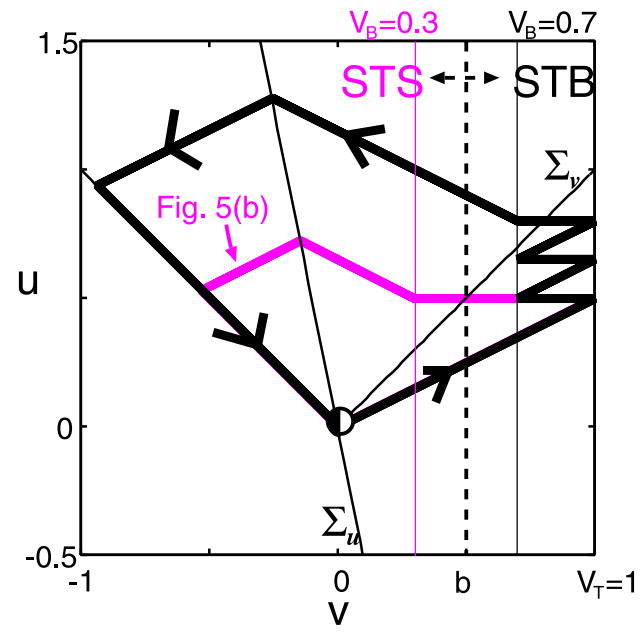

(a)

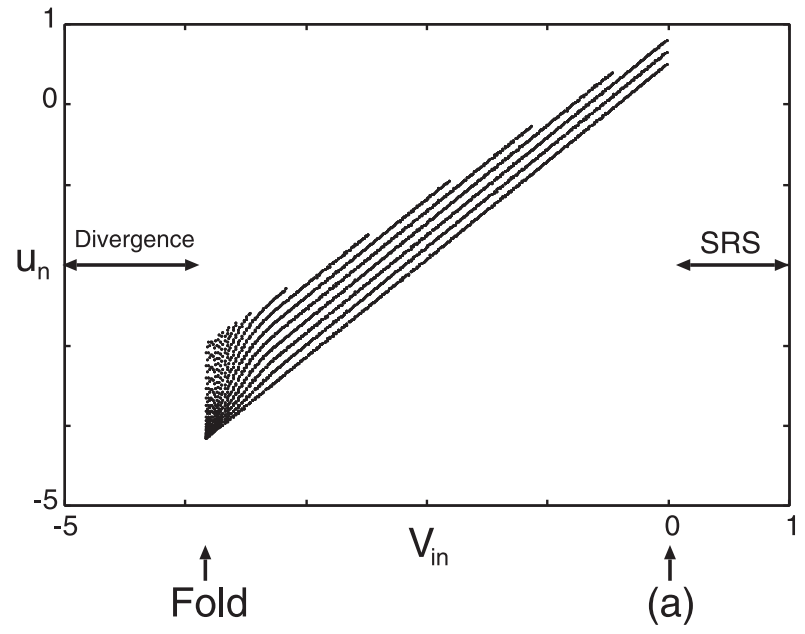

(b)

Fig. 6. The Bifurcation Scenario for $b<V_{B}$. $a=-5.0, I_{v}^{+}=1.0, I_{v}^{-}=-1.0$, $I_{u}^{+}=-0.5, I_{u}^{-}=0.5, V_{T}=1.0, V_{B}=0.7$, and $C=1.0$. (a) Stability-changing border-collision bifurcation. $V_{i n}=0.0$. (b) Bifurcation diagram. The arrows (a) and "Fold" correspond to the stability-changing border-collision bifurcation in (a) and fold limit cycle type border-collision bifurcation (not shown).

\subsubsection{Case II : $b<V_{B}$}

In this case, as shown Fig. 6, a stability-changing border-collision bifurcation causes a birth of an STB without a birth of an STS. And thus, the PWN undergoes the following bifurcation scenario.

The Bifurcation Scenario for $b<V_{B}$ :

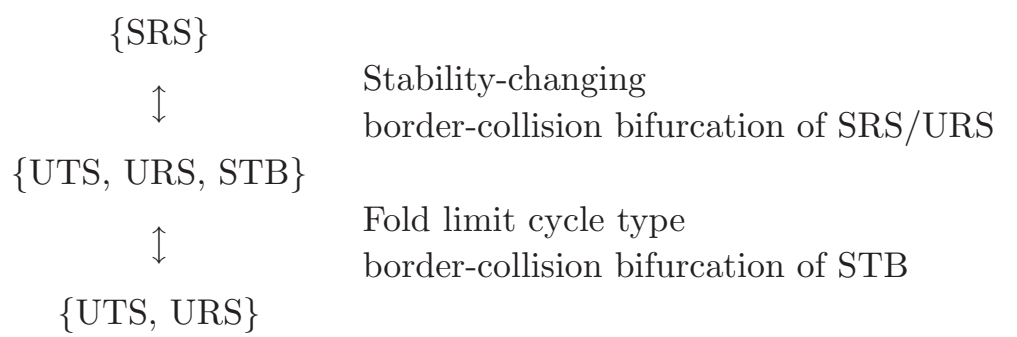

Neuron-likeness of this bifurcation scenario is discussed in the subsection 3.2.3.

\subsubsection{Neuron-likeness}

Figure 7 shows a bifurcation scenario of the Izhikevich model described by the following equation [1$3]$.

$$
\begin{aligned}
& \left\{\begin{array}{l}
\dot{v}=0.04 v^{2}+5 v+140-u+I, \\
\dot{u}=a(b v-u),
\end{array}\right. \\
& \text { if } v \geq 30 \mathrm{mV}, \text { then }\left\{\begin{array}{l}
v \leftarrow c, \\
u \leftarrow u+d,
\end{array}\right.
\end{aligned}
$$

where $v$ is a membrane potential, $u$ is a recovery variable, $(a, b, c, d)=(-0.026,-1,-57,0)$ are parameters, and $I$ is a stimulation input. The bifurcation scenario (10) of the PWN has similarities to that of the Izhikevich model as follows. Figure 7(a) shows a steady state behavior of the Izhikevich model and there exists a stable equilibrium that corresponds to the SRS in Fig. 5(a). In Fig. 7(b), the stimulation input $I$ is decreased compared to Fig. 7(a) and there exists a stable periodic orbit that corresponds to the STS in Fig. 5(c). The change of phenomena between the stable equilibrium in Fig. 7(a) and the stable periodic orbit in Fig. 7(b) is caused by a supercritical Hopf bifurcation. This change of phenomena is similar to the change of phenomena between the SRS and the URS caused by the stability-changing border-collision bifurcation in the bifurcation scenario (10) of the 

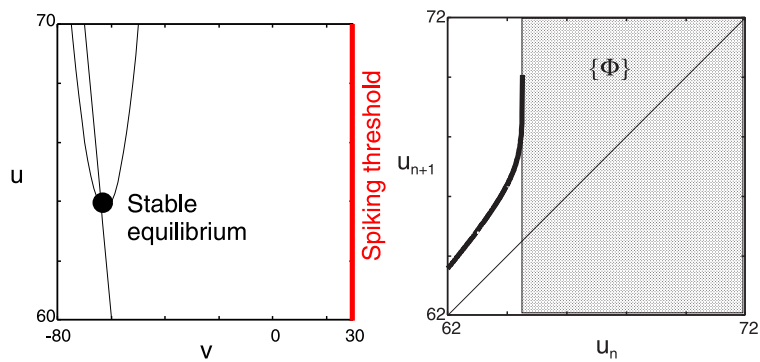

(a)
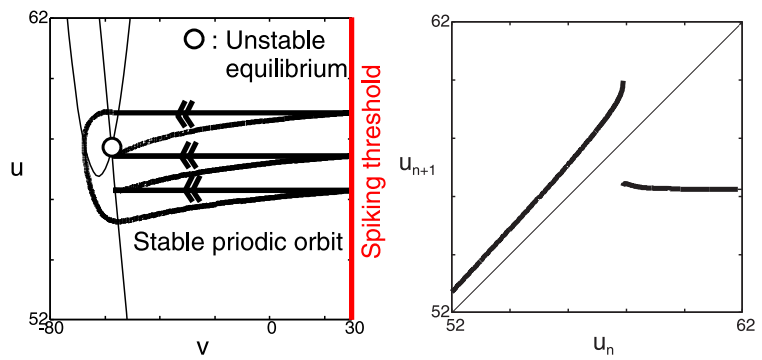

(c)

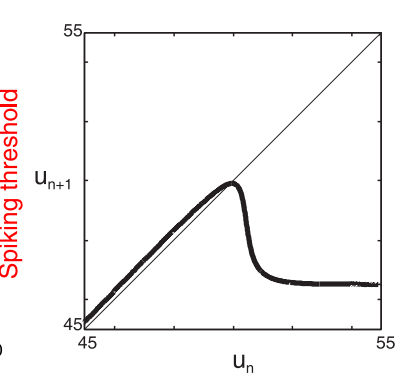

(e)

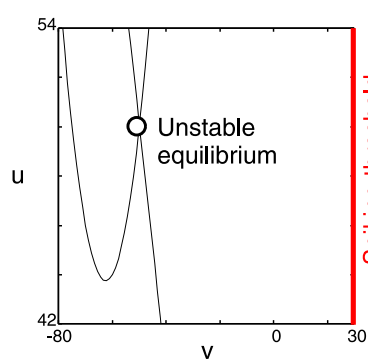

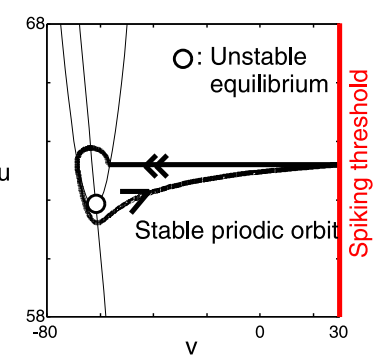

(b)

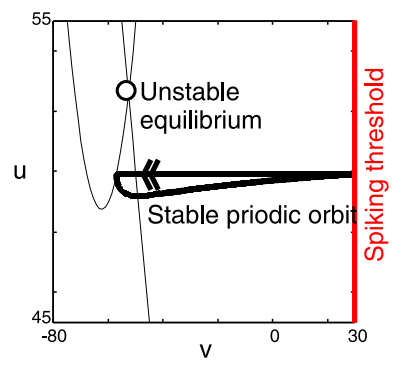

(d)
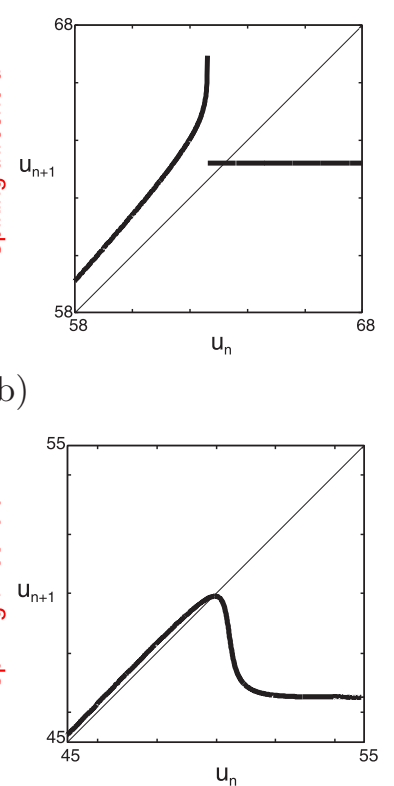

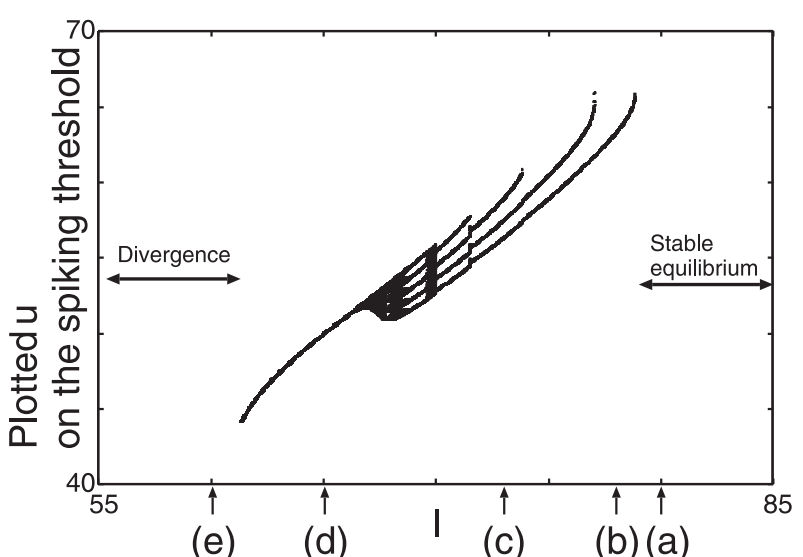

(f)

Fig. 7. A bifurcation scenario of the Izhikevich model. parameters are $a=$ $-0.026, b=-1, c=-57, d=0$. (a) Resting. $I=80$. (b) Tonic spiking. $I=78$. (c) Tonic bursting. $I=73$. (d) Tonic spiking. $I=65$ (Difference from the bifurcation scenario (10)). (e) Divergence. $I=60$. (e) Plots of the recovery variable $u$ on the spiking threshold $\{(v, u) \mid v=30 \mathrm{mV}\}$. The n-th plot of $u$ is denoted by $u_{n}$. Since $u_{n}$ uniquely determines $u_{n+1}$, a map $u_{n+1}=F_{I}\left(u_{n}\right)$, $F_{I}:(-\infty, \infty) \rightarrow(-\infty, \infty)$, can be defined like the map $F$. The arrows (a)-(e) correspond to (a)-(e), respectively.

PWN. In Fig. 7(c), the stimulation input $I$ is decreased compared to Fig. 7(b) and there exists a stable periodic orbit that corresponds to the STB in Fig. 5(d). The change of phenomena between the stable periodic orbit in Fig. 7(b) and that in Fig. 7(c) is caused by a border-collision bifurcation. This change of phenomena is similar to the change of phenomena between the STS and the STB 
caused by the border-collision bifurcation in the bifurcation scenario (10) of the PWN. On the other hand, the bifurcation scenario (10) of the PWN has some differences from those of the Izhikevich model as follows. In Fig. 7(d), the stimulation input $I$ is decreased compared to Fig. 7(c) and there exists a stable periodic orbit that corresponds to an STS of the PWN, whereas there is no change of phenomena in the bifurcation scenario (10) of the PWN that is similar to the change of phenomena between Fig. 7(c) and Fig. 7(d). In Fig. 7(e), the stimulation input $I$ is decreased compared to Fig. 7(d) and the orbit diverges. This divergence of the Izhikevich model is caused by a fall down of the map $u_{n+1}=F_{I}\left(u_{n}\right)$ below $u_{n+1}=u_{n}$, whereas the divergence of the PWN is caused by the fold limit cycle type border-collision bifurcation in the bifurcation scenario (10). The above two differences of phenomena are caused by differences of shapes of the maps between the PWN and the Izhikevich model, i.e., the map $F_{I}$ of the Izhikevich model in Fig. $7(\mathrm{~d})$ and Fig. $7(\mathrm{e})$ is continuous and less similar to the map $F$ of the PWN, whereas the map $F_{I}$ of the Izhikevich model in Fig. 7(a), Fig. 7(b) and Fig. 7(c) is relatively similar to those of PWN. In Fig. 7(f), plots the recovery variable $u$ of the Izhikevich model on the spiking threshold $\{(v, u) \mid v=30 \mathrm{mV}\}$ are shown, where the resting state in Fig. 7(a), the tonic spiking in Fig. 7(b), the tonic bursting in Fig. 7(c), the tonic spiking in Fig. 7(d), and the divergence in Fig. 7(e) are observed. Bands of chaotic behaviors are observed in Fig. 7(f) of the Izhikevich model, whereas only complex periodic orbits are observed in Fig. 5 of the PWN. This difference is also caused by differences of shapes of the maps between the PWN and the Izhikevich model, i.e., the map $F_{I}$ of the Izhikevich model has curved parts that may cause period doubling root to chaos, whereas the map $F$ of the PWN has a zero slope with discontinuity that causes period-adding bifurcations of stable periodic orbits. As a result, from a neuron's superficial response viewpoint, the PWN in Fig. 5 and the Izhikevich model in Fig7 have two major differences, i.e., existence of a stable tonic spiking near the divergence and existence of bands of chaotic behaviors (see Fig7(f)). Detailed analysis of the differences between the PWN and the Izhikevich model will be provided in our future papers. Similarly, the bifurcation scenario (11) of the PWN has similarities to a bifurcation scenario of the Izhikevich model for $(a, b, c, d)=(-0.026,-1,-45,0)$, where the detailed explanation is omitted since it is quite similar to the above explanation on the scenario (10).

\section{Implementation and measurements}

Figure 8(a) shows an implementation circuit of the GPN. The capacitor voltage $v$ (i.e., membrane potential) is applied to an absolute value circuit having a voltage follower in its input side, and the absolute value $|v|$ plus the input voltage $V_{i n}$ is applied to the positive input of the left operational transconductance amplifier (ab. OTA). Figures 8(b) and 8(c) show an implementation circuit of the absolute value circuit with the input $V_{i n}$ and characteristics of the OTA, respectively. The capacitor voltage $u$ (i.e., recovery variable) is applied to the negative input of the left OTA that outputs a current $I_{v}^{O T A}$. The left current source outputs a current $I_{v}^{O}$ and then a current $I_{v}^{O T A}+I_{v}^{O}$ flows into the right capacitor. Similarly, the right OTA outputs a current $I_{u}^{O T A}$, the right current source outputs a current $I_{u}^{O}$, and a current $I_{u}^{O T A}+I_{u}^{O}$ flows into the left capacitor. Hence, the OTAs and the current sources in Fig. 8(a) realize the VCCSs in Fig. 1(a), where $I_{v}^{+}=I_{v}^{S a t}+I_{v}^{O}, I_{v}^{-}=-I_{v}^{S a t}+I_{v}^{O}$, $I_{u}^{+}=I_{u}^{S a t}+I_{u}^{O}$, and $I_{u}^{-}=-I_{u}^{S a t}+I_{u}^{O}$. As shown in Fig. 8(a), the implementation circuit has a comparator (ab. COMP) and monostable multibivrator (ab. MM) and a switch $S$. If the membrane potential $v$ is below the reset base $V_{B}$, the switch $S$ is opened. If the membrane potential $v$ is increased but is below the spiking threshold $V_{T}$, the switch $S$ remains opened. If the membrane potential $v$ reaches the spiking threshold $V_{T}$, then the COMP triggers the MM, and the switch $S$ is closed. Hence, these components realizes the state dependent switch $S W$ in Fig. 1(a). When the switch $S$ is opened (closed), the voltage $Y$ becomes $-E(E)$. Hence, the spike-train $Y$ is generated, where $E_{H}=E$ and $E_{L}=-E$. Figure 9 depicts experimental measurements that show the two border-collision bifurcations in the previous section as follows. The change of phenomena between Fig. 9(a) and Fig. 9(b) corresponds to the stability-changing border-collision bifurcation of SRS/URS in Eq. (10). The change of phenomena between Fig. 9(b) and Fig. 9(c) corresponds to the bordercollision bifurcation of STS/STB in Eq. (10). Similarly, we have confirmed the other bifurcations 




(a)

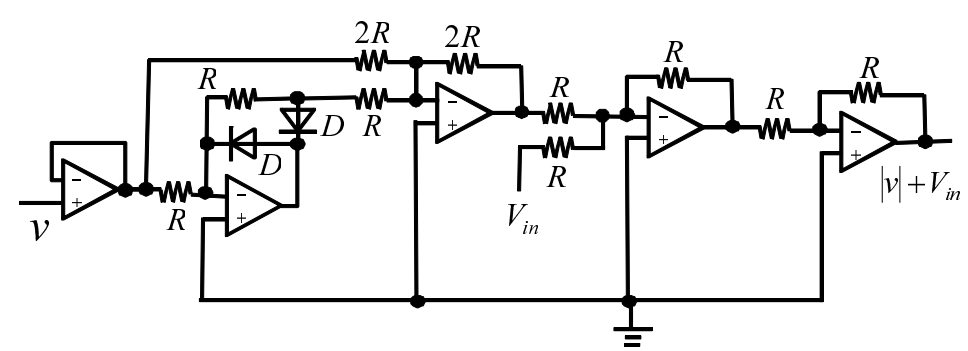

(b)

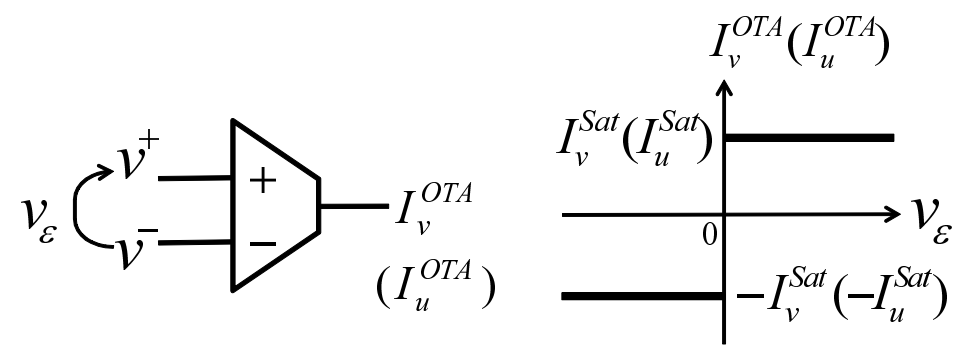

(c)

Fig. 8. (a) Implementation circuit of the GPN. (b) Absolute value circuit with the input $V_{i n}$. (c) Characteristics of an operational transconductance amplifier (ab. OTA). The GPN is implemented on a breadbord by discrete electronic components: NJM13600D (OTA and current sources), LN399 (COMP), MC14538BBCP (MM), MC14066BCP (switch), LT074 (operational amplifiers for amplifier, input voltage source, and absolute value circuit), and 1N4148 (diode for absolute value circuit).

experimentally.

\section{Concluding remarks}

The PWN has a PWC vector field with a state-dependent reset. Based on the analysis techniques for discontinuous ODEs [17], the dynamics of the inhibitory responses of the PWN can be reduced into the one-dimensional iterative map $F$ analytically. Using the map, it has been shown that the PWN can reproduce the bifurcation scenarios of the inhibitory responses, which are qualitatively similar to those of the Izhikevich model. It has been confirmed that the bifurcation scenario can be observed in the actual hardware. Future problems include: (a) analysis of more various possible bifurcation scenarios of the presented neuron model and (b) development of a neuroscience-aware network of the presented neuron model and its applications.

\section{Acknowledgments}

The authors would like to thank Professor Toshimitsu Ushio of Osaka University for valuable discussions. This work is partially supported by KAKENHI (24700225). 

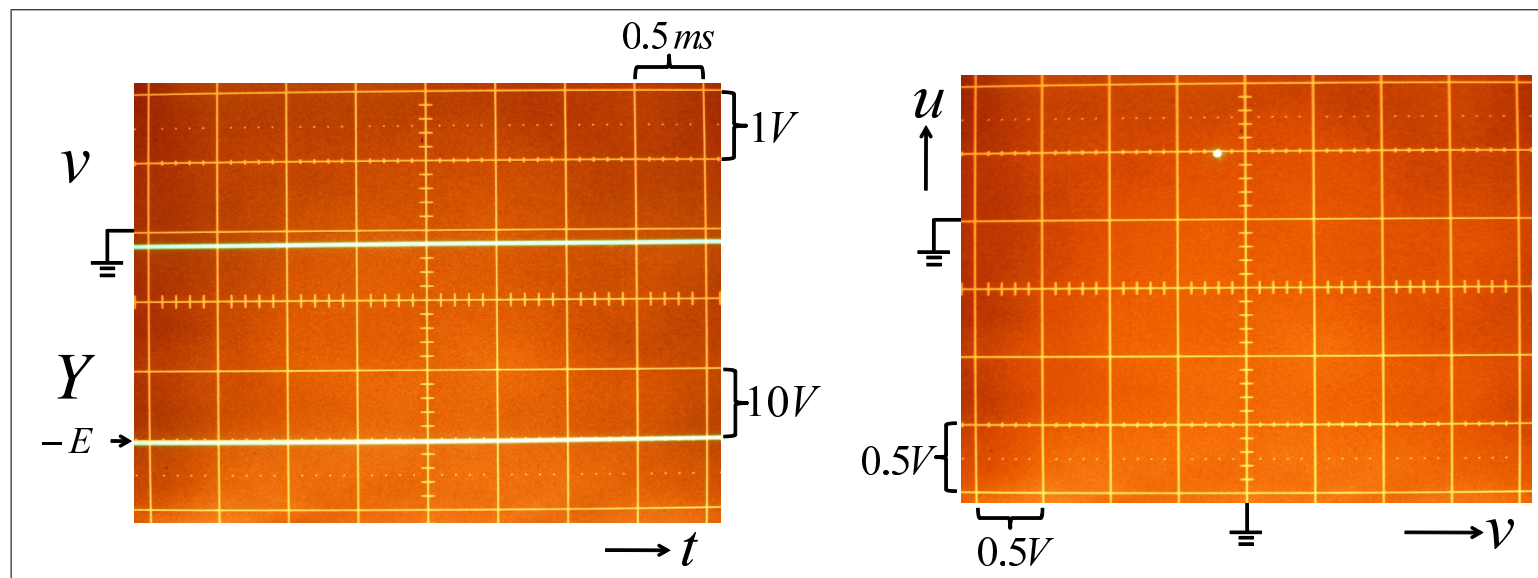

(a)

$\uparrow$ Stability-changing border-collision bifurcation of SRS/URS
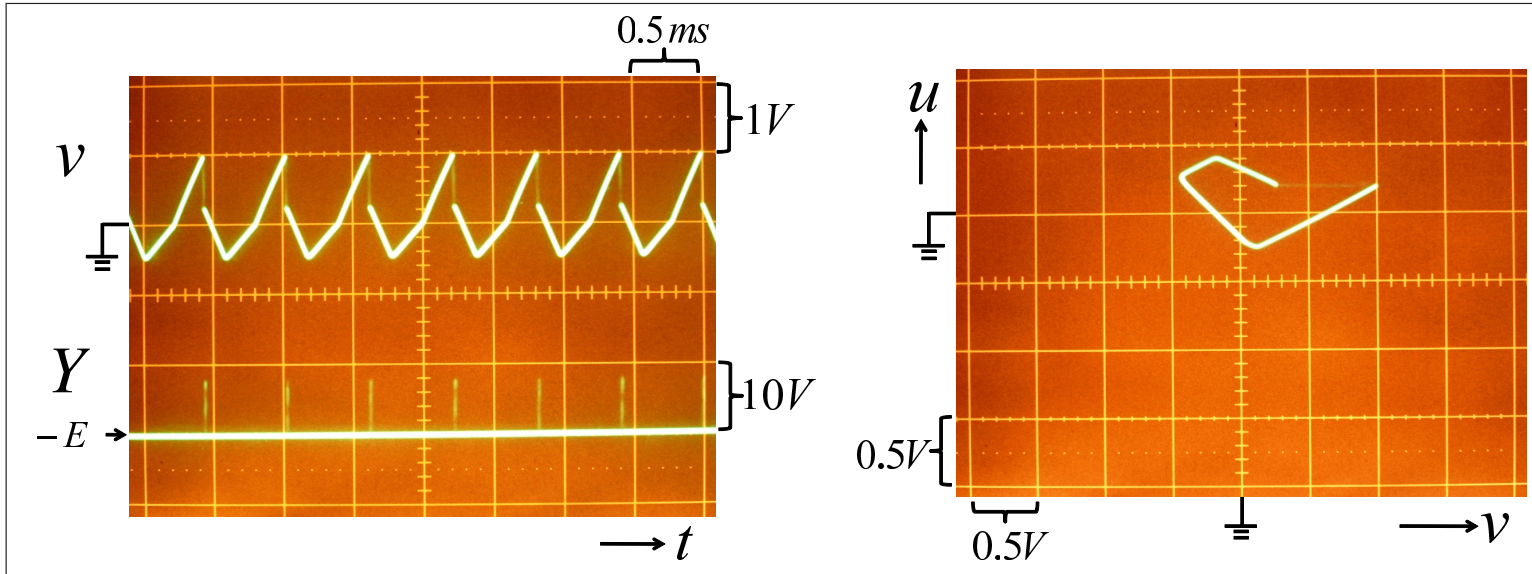

(b)

$\uparrow$ Border-collision bifurcation of STS/STB
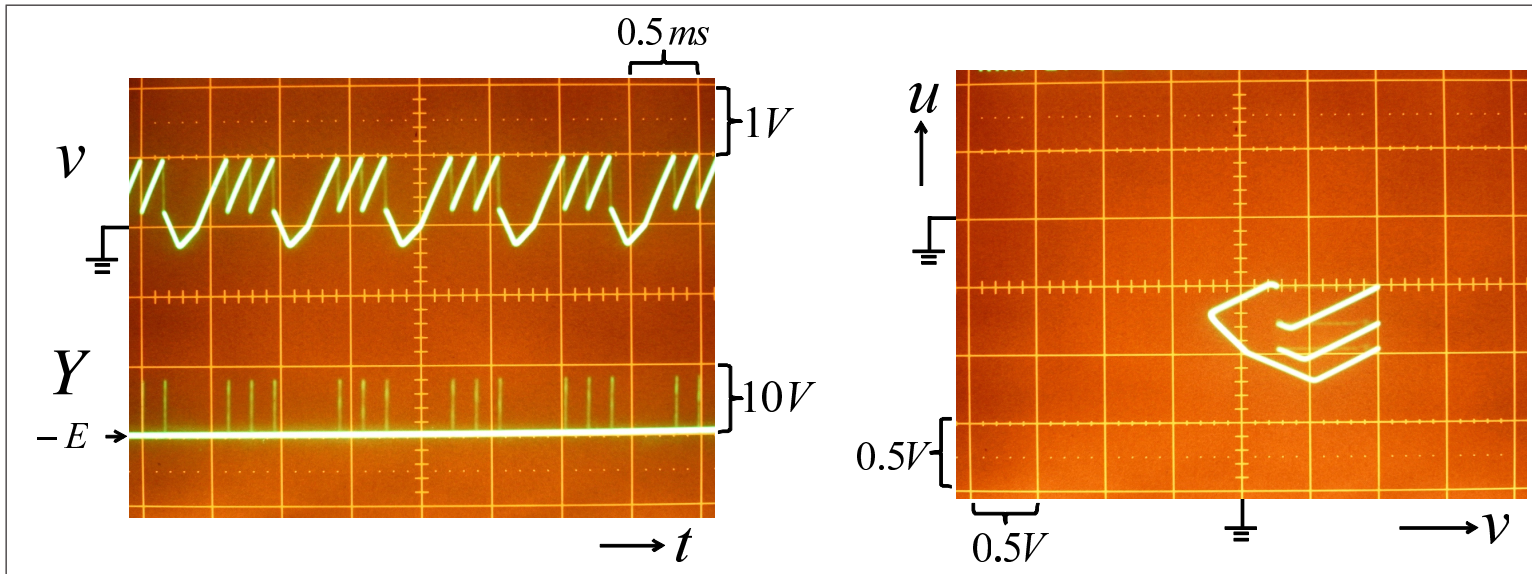

(c)

Fig. 9. Border-collision bifurcations in Eq. (10) (experimental measurements). $C \simeq 1.0 \times 10^{2}[\mathrm{nF}], a \simeq-2.5, I_{v}^{+} \simeq-I_{v}^{-} \simeq 1000[\mu \mathrm{A}], I_{u}^{+} \simeq-I_{u}^{-} \simeq$ $500[\mu \mathrm{A}], V_{T} \simeq 1.0[\mathrm{~V}]$, and $V_{B} \simeq 0.3[\mathrm{~V}]$. (a) SRS corresponding to Fig. $5(\mathrm{a})$. $V_{\text {in }} \simeq 0.3[\mathrm{~V}]$. (b) STS corresponding to Fig. $5(\mathrm{c})$. $V_{\text {in }} \simeq-0.2[\mathrm{~V}]$. (d) STB corresponding to Fig. $5(\mathrm{~d})$. $V_{i n} \simeq-1.0[\mathrm{~V}]$. 


\section{References}

[1] E. Izhikevich, Dynamical systems in neuroscience: The geometry of excitability and bursting, The MIT press, 2007.

[2] E. Izhikevich, "Which model to use for cortical spiking neurons?," IEEE Trans. Neural Netw., vol. 15, no. 5, pp. 1063-1070, 2004.

[3] E. Izhikevich, "Simple model of spiking neurons," IEEE Trans. Neural Netw., vol. 14, no. 6, pp. 1569-1572, 2004.

[4] A. Hodgkin and A. Huxley, "A quantitative description of membrane current and its application to conduction and excitation in nerve," The Journal of physiology, vol. 117, no. 4, pp. 500-544, 1952.

[5] G. Indiveri et al., "A VLSI array of low-power spiking neurons and bistable synapses with spiketiming dependent plasticity," IEEE Trans. Neural Netw., vol. 17, no. 1, pp. 211-221, 2006.

[6] T. Yu and G. Cauwenberghs, "Analog VLSI biophysical neurons and synapses with programmable membrane channel kinetics," IEEE Trans. Biomed. Circuits Syst., vol. 4, no. 3, pp. 139-148, 2010.

[7] T. Asai et al., "A subthreshold MOS neuron circuit based on the Volterra system," IEEE Trans. Neural Netw., vol. 14, no. 5, pp. 1308-1312, 2003.

[8] E. Farquhar and P. Hasler, "A bio-physically inspired silicon neuron," IEEE Trans. CAS-I, vol. 52, no. 3, pp. 477-488, 2005.

[9] A. Basu and P. Hasler, "Nullcline-Based Design of a Silicon Neuron," IEEE Trans. CAS-I, vol. 57 , no. 11, pp. 2938-2947, 2010.

[10] M. Simoni and S. DeWeerth, "Adaptation in a VLSI model of a neuron," IEEE Trans. CAS-II, vol. 46, no. 7, pp. 967-970, 2002.

[11] C. Rasche, "An a VLSI basis for dendritic adaptation," IEEE Trans. CAS-II, vol. 48, no. 6, pp. 600-605, 2001.

[12] T. Kohno and K. Aihara, "A MOSFET-based model of a class 2 nerve membrane," IEEE Trans. Neural Netw., vol. 16, no. 3, pp. 754-773, 2005.

[13] N. Mizoguchi, Y. Nagamatsu, K. Aihara, and T. Kohno, "A two-variable silicon neuron circuit based on the izhikevich model," Artificial Life and Robotics, vol. 16, no. 3, pp. 383-388, 2011.

[14] Y. Yamashita and H. Torikai, "A novel PWC spiking neuron model: Neuron-like bifurcation scenarios and responses," IEEE Trans. CAS-I (to appear).

[15] Y. Yamashita and H. Torikai, "Bursting analysis and synapse mechanism of a piece-wise constant spiking neuron model," Proc. IEEE-INNS/IJCNN, pp.193-200, 2012.

[16] Y. Yamashita and H. Torikai, "A generalized PWC spiking neuron model and its neuron-like activities and burst-related bifurcations," IEICE Trans. Fund., vol.E95-A, no.7, pp.1156-1163, 2012.

[17] M. Di Bernardo, Piecewise-smooth dynamical systems: theory and applications, Springer, 2008.

[18] Y. Matsuoka et al., "Chaotic spike-train with line-like spectrum," IEICE Trans. Fund., vol. E92A, no. 4, pp. 1142-1147, 2009.

[19] Y. Matsuoka and T. Saito, "A simple chaotic spiking oscillator having piecewise constant characteristics," IEICE Trans. Fund., vol. E89-A, no. 9, pp. 2437-2440, 2006.

[20] T. Tsubone and T. Saito, "Manifold piecewise constant systems and chaos," IEICE Trans. Fund., vol. E82-A, no. 8, pp. 1619-1626, 1999.

[21] I. Vida et al., "Shunting inhibition improves robustness of gamma oscillations in hippocampal interneuron networks by homogenizing firing rates," Neuron, vol. 49, no. 1, pp. 107-117, 2006. 